\title{
The impact of factors in work environment (especially shift and night work) on neoplasia of female reproductive organs
}

\author{
Bartosz Bilski', Paweł Rzymski², Katarzyna Tomczyk², Izabela Rzymska ${ }^{3}$ \\ ${ }^{1}$ Department of Preventive Medicine, Poznan University of Medical Sciences, 11 Smoluchowskiego Str., Poznan, Poland, \\ phone: +48 61 8612243, e-mail: Bilski@ump.edu.pl \\ 2 Department of Mother's and Child's Health, Poznan University of Medical Sciences, Gynecologic and Obstetrical \\ University Hospital, 33 Polna Str., 60-535 Poznan, Poland \\ 3 Department of Social Sciences, Poznan University of Medical Sciences, 79 Dąbrowskiego Str., 60-529 Poznan, Poland
}

\begin{abstract}
Shift work, due to disruption of circadian rhythms, can interfere with a number of physiological functions. It may lead to multiple pathologies (functional gastrointestinal disorders, peptic ulcer disease, hormonal disorders including impaired melatonin secretion, cardiovascular disease, mental disabilities, neurological disorders etc.). In the last few years, we started to think about the association between disruption in melatonin secretion and the occurrence of certain malignancies. Authors describe and discuss pathophysiology, epidemiological and clinical data concerning influence of shift work to occurrence of some neoplasms.
\end{abstract}

Keywords: neoplasm, shift work, melatonin.

\section{Introduction}

Shift work, due to disruption of circadian rhythms, can interfere with a number of physiological functions. It may lead to multiple pathologies (functional gastrointestinal disorders, peptic ulcer disease, hormonal disorders - including impaired melatonin secretion, cardiovascular disease, mental disabilities, neurological disorders etc.). In the last few years, we started to think about the association between disruption in melatonin secretion and the occurrence of certain malignancies. There are confirming epidemiologic data from almost seventy years before [1]. Melatonin blood level fluctuations may be a factor participating in the development of especially breast and colorectal cancer. Moreover, it seems that the hormone has also an impact on ovary, endometrium, and prostate neoplasia occurrence. There is also a suspicion that disturbances in the melatonin secretion may be a factor inducing changes in the physiology of the mucous membranes in gastrointestinal tract (ulcers), hypertension and abnormal irregular menstrual cycle - frequent diseases in shift workers [1].

\section{Physiology, pathophysiology and results from experimental studies}

Suprachiasmatic nucleus, situated in the anterior part of hypothalamus, is one of the most important parts of the brain (as well as medulla oblongata, pons and raphe nuclei), affecting the onset of mammalian twenty-four hour rhythm series in physiological functions [2]. Alteration in circadian rhythm causes destruction just in the anterior part of hypothalamus in experimental animals. The optic fibres from the retina reach these structures, and by stimulus may modify the daily rhythm. Alteration in daily rhythm of melatonin secretion by the pineal gland is the consequence of their function. It was found that a significant impact on 
the retina of the human eye (including the synthesis of melatonin) has exposure to light with a wavelength between 446 and $484 \mathrm{~nm}$ [3]. Light, of less than 1 lux significantly inhibits the synthesis of nocturnal melatonin [3]. Melatonin is produced in a significant amount in the pineal gland. Amino acid L-tryptophan is the substrate for this synthesis. Its secretion by the pineal gland depends on the circadian rhythm, closely related to the changes of the amount of light reaching the retina during the day and at night, increases in darkness and decreases during exposure to natural or artificial light. Increased melatonin production is associated with longer night periods - in an annual rhythm and in higher latitudes. Melatonin allows for organisms adaptation to changes in lighting rhythm. Among commonly known properties of this substance one can distinguish the function of antioxidants, adjusting sleep-wake rhythm, immunomodulatory function, effects on puberty and the process of reproduction, the emergence of mental disorders and diseases of the central nervous system [4]. Furthermore, melatonin has a positive influence on fight against Gram-negative bacterias infections by immune system, reduces the immunosuppressive effects of stress, enhances antitumor activity of interleukin-2 (IL-2), interleukin-6 (IL-6), interleukin-12 (IL-12) interferon (INF-y) and increases level of neutral killers (NK) $[4,5]$. It is known, that this substance may be a diagnostic and prognostic marker of neoplasia. In addition, melatonin heals circadian rhythm alteration caused by air travel (jet lag) and shift work [4].

Melatonin is also produced in rich in serotonin enterochromaffine cells (APUD) in the gastrointestinal tract. It gets to the portal circulation (endocrine effect) and lymphatic tissue but has also auto- and paracrine effect. Thus, it affects the function of mucosa and reduces the voltage of smooth muscle of digestive tract. The presence of food, not light stimulation of the retina is the stimulus for melatonin secretion in the gastrointestinal tract [5]. It has been shown that a colon cancer development is associated with decrease of APUD cells number, that synthesize serotonin, melatonin and other peptides [6, 7].

Melatonin may participate in the process of neoplasia in female reproductive system through various ways [9]:

- melatonin has anti-estrogenic properties - reduces estrogen secretion by the ovaries,

There are an evidence that suppresion of the hypothalamus-pituitary-ovarian axis reduces $\mathrm{LH}$ and 17betha estradiol [10]. Deteriorated levels of mela- tonin during night-work cause an increased level of estrogen in premenopausal women [11].

- melatonin stimulates the synthesis of progestagens It seems that via an impact on the transcriptional activity of the steroidogenic enzymes melatonin may modulate ovarian theca cell steroidogenesis at the molecular level $[12,13]$.

- the occurrence of seasonal variability in, e.g. endometrial hyperplasia can be associated with melatonin levels.

According to Dznelashvili (2013) received results, the more complicated the type of endometrial hyperplasia is, the more consistently melatonin is reduced in blood plasma [14].

- melatonin levels decrease rapidly during menopause period - typical period of breast and endometrium cancers occurrence,

Melatonin therapy in menopausal patient causes the decrease of LH and FSH level in the blood and this therapy is under intensive investigation as a kind of pineal-pituitary-ovarian axis control $[15,16]$.

- obesity is often associated with cancer - such persons have more frequent disturbed daily melatonin levels,

Significant finding is the induction by melatonin of white adipose tissue browning, which may be related to its effects against oxidative stress as well as body weight reduction in experimental animals [17].

- diabetes, which is correlated with the occurrence of certain cancers (e.g. endometrial cancer), causes reducing in secretion of melatonin by the pineal gland and increases the likelihood of calcification of this gland $[10,17]$.

For example the mean of salivary melatonin level was significantly lower in patients with type II diabetes [18].

During in vitro treatment, it was found, that melatonin inhibits the process of angiogenesis in tumors (decreases the expression of VEGF receptor and increases the expression of epidermal growth factor receptor and insulin growth factor-1), and significantly affects the rate of cell's DNA synthesis in some tumors [19, 20]. As it was mentioned, it has been shown that it may prevent tumor development by enhancement of immune-response: it stimulates proliferation and maturation of immune cells (NK, T/B- lymphocytes, granulocytes monocytes) [20]. In vitro, melatonin significantly inhibits tumor growth of endometrial, stomach and adrenals cancer, and in some cases of renal, colon and 
rectum cancer. In clinical experimental studies on rats, melatonin inhibited the increase of some form of prostate adenocarcinoma [20]. The relationship has been shown between the growth rates of implanted human breast cancer cells in rats (MCF-7) and the rhythm of illumination. The study evaluated melatonin and linolenic acid levels in the two groups of rats - first, which lived alternately twelve hours in light and darkness, and the other exposed to light all day and night. In rats that were exposed to artificial lighting all the time lower concentrations of melatonin levels was found and rapid proliferation of cancer. The proposed mechanism for melatonin, which limits the growth of the tumor is inhibition of the cellular receptors of tumor's cells, thus affecting the metabolic linolenic acid utilization. Another study revealed that melatonin has directly inhibitory effects on MCF7 human breast cancer cell growth in culture, although supra- or subphysiological levels of melatonin are completely ineffective [21]. Also precursors and metabolites of melatonin such as serotonin, $\mathrm{N}$-acetylserotonin and 6-hydroxymelatonin do not inhibit MCF-7 cell growth. It seems that the antiproliferative effect of melatonin may be dependent on the presence of serum and a complex interaction with hormones such as estradiol and/or prolactin because without it melatonin loses antimetabolic function [21]. In terms of breast cancer, population-based case-control study suggests that polymorphisms in circadian genes and melatonin's biosynthesis genes (like CLOCK, MTNR1B, NPAS2 and ARNTL) may be involved in the process of neoplasia [21].

In case of endometrial cancer, the cytostatic effect of melatonin seems to be mediated by melatonin receptor 1 (MT1) but not MT2, and attenuation of estrogen receptor alpha (ER alpha) expression in endometrial cancer cells [22].

Other experimental studies also confirm anticarcinogenic impact of that substance - eg. the administration of melatonin in rats with removed pineal gland results in inhibition of carcinogenesis [23]. It has been found in experimental studies in rats that changes in circadian rhythm significant disturb the functioning of the immune system (inter alia characterized by changes in the blood results) and increase the risk of tumor growth. Melatonin reduced the risk of such effects [23] and we can conclude it has immuno-modulating, anti-carcinogenic, antiproliferative and anti-inflammatory properties.

Interesting study of Qin et al. (2012) has shown one of the possible anti-proliferative and anticarcinogenic mechanism of melatonin. It reveals, that the hormone may reduce the levels of MMP9 (matrix metalloproteinases) mRNA and protein through up-regulation of TIMP1 (MMP9-natural inhibitor) mRNA and protein, via the nuclear factor $\mathrm{kB}$ translocation (NFkB/p65) as well as through direct mechanism: inhibition of MMP9 's activity by binding to its active side. MMPs induced by inflammatory cytokines IL1 $\beta$ might be a potential mechanism that affects endothelial barrier function, so that might be associated with tumor invasion, metastasis, and angiogenesis [24].

The anti-inflammatory mechanism has been also examined. Melatonin inhibits lipopolysaccharide (LPS)-induced cyclooxygenase-2 (COX-2) and inducible nitric oxide synthase (iNOS) protein levels via inhibition of p300 histone acetyltransferase (p300 HAT) activity and p52 acetylation. Interestingly, in experimental studies, some carcinogens eg. 7.12-dimethyl- [a] anthracene (DMBA) caused growth of the maximum level of melatonin present in the blood during the night, which could indicate a physiological mechanism, which enables the synthesis of melatonin in the case of significant exposure to the carcinogen. In experimental studies in rodents, it was found that other peptides of the pineal gland (epitalamina) and similar synthetic tetrapeptide - epitalon (Ala-Glu-Asp-Gly) are potential inhibitors of carcinogenesis in breast cancer [25].

\section{Clinical and epidemiological data}

Shift work affects daily level of melatonin in the blood as well as certain hormones participating in the process of carcinogenesis. Three years lasting research on the relationship between labor rhythm and expulsion of melatonin metabolite in morning urine (6-sulfatoxymelatonin) and levels of steroid hormones in plasma, in 80 women before menopause, showed an increased levels of serum estradiol. It concerned women who have been employed in shift work for at least fifteen years (mean concentration of serum estradiol $10.1 \mathrm{pg} /$ $\mathrm{ml}$ ) compared with those who have never worked like that $(8.8 \mathrm{pg} / \mathrm{ml})(\mathrm{p}=0.03)$. It revealed a statistically significant inverse relationship between the number of worked nights within two weeks and the concentration of melatonin metabolite in urine $(r=-0.30, p=$ 0.008 [26]. Melatonin seems to have anti-estrogenic and anti-aromatase activity as well as may affect fat metabolism, which are risk factors of endometrial cancer. Viswanathan et al. conducted a study consisting of the 121,701 women, where 53,487 had night shift work and 515 of them developed invasive endometrial cancer. The study revealed that women who worked 
$20+$ years of rotating night shifts had a significantly increased risk of endometrial cancer (multivariate relative risks MVRR: 1.47) and obese women doubled their baseline risk of endometrial cancer (MVRR, 2.09) [27]. Because of the fact that darkness increases the plasma concentrations of melatonin (treated as an anti-cancer substance), epidemiological studies were conducted among populations without the effect of light on melatonin secretion - the blind and visually impaired. In the Swedish study, 1567 completely blind and 13292 visually impaired persons were found to have much lower risk of cancer ( $\mathrm{RR}=0.69 ; 95 \% \mathrm{Cl}=0.59-0.82$ ) respectively to general population [27]. It was also revealed that the average concentration of melatonin in the blood is significantly lower in patients with prostate and breast cancer [28]. Epidemiological studies in the population of 78.586 women (nurses) working in shifts for 14 years (at least three night shifts a month), comparing to women who have never been working in shifts, showed that the risk of colorectal cancer was not increased (RR = 1.00; 95\% Cl: 0.84-1.19). Whereas, in the analysed population the risk was definitely growing on average by $35 \%(95 \% \mathrm{Cl}=1.3-1.77)$, after working more than 15 years in the night-shift system [29]. In the same population, the risk of breast cancer grew moderately with the time of shift work (at least 3 night shifts a month). The risk of breast cancer grew in population with the work experience from one to 29 years for about $8 \%(95 \% \mathrm{Cl}=0.99-1.18$ in the population with work experience from one year to 14 years and 0.90-1.30 in the population with experience of 15 to 29 years). Only long-term shift work (above 30 years) caused the risk significantly increased on average by $36 \%(95 \% \mathrm{Cl}=1.04-1.78)$ [29]. However, other study of Schernhammer et al. showed that already more than 20 years of rotating night shift work was related with elevated risk (30). When it comes to breast cancer Hansen examined also nurses and revealed confirming data: nurses who worked rotating shifts after midnight had a significantly increased odds ratio $O R$ (1.8; $\mathrm{Cl} 1.2-2.8)$ for breast cancer compared to nurses with permanent day work. No association was found in a small group of nurses with evening work and without night work (OR $=0.9 ; 0.4-1.9)$ [31]. Jia et al. in metaanalysis of epidemiological studies confirmed association between night shift work and breast cancer. Nevertheless, Kamdar et al. in their meta-analysis conclude that there is weak evidence to support previous reports. Using random-effects models, the pooled relative risk (RR) for individuals with ever night-shift work exposure was 1.21 , for short-term night-shift workers ( $<8$ years) it was 1.13 and for long-term night-shift workers ( $\geqslant 8$ years) it was 1.04 [32]. Clinical case control study in Seattle analysed night habits at work of 813 women with breast cancer and 793 women as a group control. The study showed that increased by an average of $14 \%$ breast cancer risk applies to people who often do not sleep at night in the 10 years prior to the analysis $(95 \% \mathrm{Cl}=1.011 .28)$. The risk of this disease did not grow in the case of people who usually have often intermittent sleep with lightening artificial light [33]. In relation to ovarian cancer, large prospective study of 181.548 women revealed no association between duration of rotating night shift work and risk of ovarian cancer [34].

\section{Other occupational factors affecting level of melatonin}

One of the professional factor affecting melatonin secretion is exposure to electromagnetic fields. There have been a number of studies attempting to correlate the relationship between the effects of exposure to this factor and the epidemiology of breast cancer [35]. Most of these studies have not shown any strong relationship between these factors. Future studies should focus on a comprehensive assessment of the impact and interdependence of different exposure parameters, field frequency, and co-occurrence of shift work and the individual factors like age and hormonal factors (eg. estrogen receptors). Norwegian study of 2619 women is an example of a significant impact of occupational exposure to electromagnetic fields and simultaneous shift work on carcinogenesis. The relative risk for carcinogenesis in this population was significantly increased by $20 \%$ compared to the general population, and in the case of breast cancer increased by $50 \%$ ! [35].

\section{Melatonin in treatment}

There is some evidence that high-dose of melatonin may be beneficial in the combined cancer's treatment (e.g. with chemotherapy). Regression of tumor mass was described for breast cancer and prostate cancer but also lung, kidney, liver, pancreatic, stomach and colon cancer. Worth noticed is the conclusion from the Lissoni's study that melatonin plus chemotherapy in patients with metastatic solid tumors seems to increase regression rate and one-year survival rate by approximately 50\% compared to chemotherapy alone. Lung 
cancer - 104 cases, breast cancer - 77, gastrointestinal tract neoplasms- 42 and head and neck cancers27 were taken into consideration. Another thing is that melatonin seems to reduce side effect of chemotherapy. For example, it is proven to enhance platelet number in patients with thrombocytopenia due to chemotherapy of metastatic breast cancer $[7,36]$. It has an impact on other haematopoietic cells as well as may diminish neurotoxicity, cardiotoxicity, stomatitis and asthenia [36].

\section{Summary}

Circadian rhythm disorders caused by work in the night work shift system seems to be the interesting issue in practical occupational medicine. It requires further detailed epidemiological studies and clinical trials. Please note that, for mentioned cancers, there are many other, highly significant risk factors. It is worth noting, that there is a higher risk of a malignant tumor occurrence while working in shifts for many years, especially when there are other unresponsive risk factors (eg. age, genetic factors) and customisable ones. Practically, the exogenous supplementation of melatonin seems to be important in reducing the negative shift work's impact $[32,37]$. Interesting would be the analysis of the effectiveness of such therapy in relation to other health problems associated with such a system work, especially because recent studies showed different activity of melatonin transcription in endometrial cancer. It could provide new diagnostic and prognostic markers od the disease [37].

\section{Acknowledgements}

\section{Conflict of interest statement}

The authors declare that there is no conflict of interest in the authorship or publication of contribution.

\section{Funding sources}

There are no sources of funding to declare.

\section{References}

1. Karasek M, Pawlikowski M. Pineal gland, melatonin and cancer. Review. Neuroendocrinol Lett, 1999;20(34):139-144.

2. Schernhammer ES, Schulmeister K. Melatonin and cancer risk: does light at night compromise physiologic cancer protection by lowering serum melatonin levels? Br J Cancer. 2004;90:941-943.

3. Glickman G, Levin R, Brainard GC. Ocular input for human melatonin regulation: relevance to breast cancer. Neuroendocrinol Lett. 2002;Supl 2:17-22.

4. Maestroni GJ. The immunotherapeutic potential of melatonin. Expert Opin Investing Drugs. 2001;10:467-476.
5. Srinivasan V, Spence DW, Pandi-Perumal SR, Trakht I, Cardinali DP. Therapeutic actions of melatonin in cancer: possible mechanisms. Integr Cancer Ther. 2008;7:189203.

6. Bubenik GA, Blask DE, Brown GM, Maestroni GJ, Pang SF, Reiter RJ. Prospects of the clinical utilization of melatonin. Biol Signals Recept. 1998;7:195-219.

7. Bubenik GA. Localization, physiological significance and possible clinical implication of gastrointestinal melatonin. Biol Signals Recept. 2001;10:350-366.

8. Kozlova IV, Osadchuk MA, Kvetnoi IM. Changes in the APUD system of the large intestine as a risk factor for colorectal cancer. Klin Med. 1999;7:26-29.

9. Sandyk R, Anastasiadis PG, Anninos PA, Tsagas N. Is the pineal gland involved in the pathogenesis of endometrial carcinoma. Int J Neurosci. 1992;62:89-96.

10. Chuffa LG, Seiva FR, Fávaro WJ, Teixeira GR, Amorim JP, Mendes LO et al. Melatonin reduces LH, 17 beta-estradiol and induces differential regulation of sex steroid receptors in reproductive tissues during rat ovulation. Reprod Biol Endocrinol. 2011;2:9:108.

11. Stevens RG, Schernhammer E. Epidemiology of urinary melatonin in women and its relation to other hormones and night work. Cancer Epidemiol Biomarkers Prev. 2005; 14:551.

12. Tanavde VS, Maitra A. In vitro modulation of steroidogenesis and gene expression by melatonin: a study with porcine antral follicles. Endocr Res. 2003;29:399-410.

13. Maganhin CC, Simões RS, Fuchs LF, Sasso GR, Simões MJ, Baracat EC, Soares JM Jr. Melatonin influences on steroidogenic gene expression in the ovary of pinealectomized rats. Fertil Steril. 2014;102:291-8.

14. Dznelashvili NO, Kasradze DG, Tavartkiladze AG, Mariamidze AG, Dzhinchveladze DN. Expression of epidermal growth factor receptor and plasmatic level of melatonin in simple and complexendometrial hyperplasia. Georgian Med News. 2013;223:91-5.

15. Bellipanni G, Bianchi P, Pierpaoli W, Bulian D, Ilyia E. Effects of melatonin in perimenopausal and menopausal women: a randomized and placebo controlled study. Exp Gerontol. 2001;36:297-310.

16. Diaz BL, Llaneza PC. Endocrine regulation of the course of menopause by oral melatonin: first case report. Menopause. 2008;15:388-92.

17. Navarro-Alarcón M1, Ruiz-Ojeda FJ, Blanca-Herrera RM, A-Serrano MM, Acuña Castroviejo D, Fernández-Vázquez G, Agil A. Melatonin and metabolic regulation: a review. Food Funct. 2014;5:2806-32.

18. Abdolsamadi H, Goodarzi MT, Ahmadi Motemayel F, Jazaeri M, Feradmal J, Zarabadi M, Hoseyni $M$ et al. Reduction of Melatonin Level in Patients with Type II Diabetes and Periodontal Diseases. J Dent Res Dent Clin Dent Prospects. 2014;8:160-5.

19. Lissoni P, Rovelli F, Malugani F, Bucovec R, Conti A, Maestroni GJ. Anti-angiogenic activity of melatonin in advanced cancer patients. Neuroendocrinol Lett. 2001;22:4547.

20. Miller SC, Pandi-Perumal SR, Esquifino Al, Cardinali DP, Maestroni GJ. The role of melatonin in immuno-enhancement: potential application in cancer. Int J Exp Pathol. 2006;87:81-7. 
21. Rabstein S, Harth V, Justenhoven C, Pesch B, Plöttner S, Heinze E, Lotz A, Baisch C, Schiffermann M, Brauch $\mathrm{H}$, Hamann U, Ko Y, Brüning T; on behalf of the GENICA Consortium. Polymorphisms in circadian genes, night work and breast cancer: Results from the GENICA study. Chronobiol Int. 2014;17:1-8.

22. Watanabe M, Kobayashi Y, Takahashi N, Kiguchi K, Ishizuka B. Expression of melatonin receptor (MT1) and interaction between melatonin and estrogen in endometrial cancercell line. J Obstet Gynaecol Res. 2008;34:567-73.

23. Anisimov VN. The light-dark regimen and cancer development. Neuroendocrinol Lett. 2002;Supl 2:28-36.

24. Qin W, Lu W, Li H, Yuan X, Li B, Zhang Q, Xiu R. Melatonin inhibits IL1 $\beta$-induced MMP9 expression and activity in human umbilical vein endothelial cells by suppressing NFKB activation. J Endocrinol. 2012;214:145-53.

25. Deng WG, Tang ST, Tseng HP, Wu KK. Melatonin suppresses macrophage cyclooxygenase-2 and inducible nitric oxide synthase expression by inhibiting p52 acetylation and binding. Blood. 2006;108:518-24.

26. Schernhammer ES, Rosner B, Willett WC, Laden F, Colditz GA, Hankinson SE. Epidemiology of urinary melatonin in women and its relation to other hormones and night work. Cancer Epidemiol Biomarkers Prev. 2004;13: 936-943.

27. Viswanathan AN, Hankinson SE, Schernhammer ES. Night shift work and the risk of endometrial cancer. Cancer Res. 2007;67:10618-22.

28. Oosthuizen JM, Bornman MS, Barnard HC, Schulenburg GW, Boomker D, Reif S. Melatonin and steroid-dependent carcinomas. Andrologia. 1989;21:429-431.

29. Schernhammer ES, Laden F, Speizer FE, Willett WC, Hunter DJ, Kawachi I et al. Night-shift work and risk of colorectal cancer in the nurses' health study. J Natl Cancer Inst. 2003;95:825-828.

30. Schernhammer ES, Kroenke $\mathrm{CH}$, Laden $\mathrm{F}$, Hankinson SE. Night work and risk of breast cancer. Epidemiology. 2006;17:108-11.

31. Hansen J, Stevens RG. Case-control study of shift-work and breast cancer risk in Danish nurses: impact of shift systems. Eur J Cancer. 2012;11:1722-9.
32. Kamdar BB, Tergas Al, Mateen FJ, Bhayani NH, Oh J. Night-shift work and risk of breast cancer: a systematic review and meta-analysis. Breast Cancer Res Treat. 2013;138:291-301.

33. Davis S, Mirick DK, Stevens RG. Night shift work, light at night, and risk of breast cancer. J Natl Cancer Inst. 2001;93:1557-1562.

34. Poole EM, Schernhammer ES, Tworoger SS. Rotating night shift work and risk of ovarian cancer. Cancer Epidemiol Biomarkers Prev. 2011;20:934-8.

35. Tynes T, Hannevik M, Andersen A, Vistnes Al, Haldorsen $T$. Incidence of breast cancer in Norwegian female radio and telegraph operators. Cancer Causes Control. 1996; 2:197-204.

36. Sanchez-Barcelo EJ, Mediavilla MD, Alonso-Gonzalez C, Reiter RJ. Melatonin uses in oncology: breast cancer prevention and reduction of the side effects of chemotherapy and radiation. Expert Opin Investig Drugs. 2012;21:6, 819-31.

37. Witek $A$, Jęda W, Baliś $M$ et al. Expression of melatonin receptors genes and genes associated with regulation of their activity in endometrial cancer. Gin Pol. 2015;4:248255.

Acceptance for editing: 2015-11-10 Acceptance for publication: 2015-12-31

Correspondence address: Bartosz Bilski Department of Preventive Medicine Poznan University of Medical Sciences 11 Smoluchowskiego Str., Poznan, Poland phone: +48618612243 email: Bilski@ump.edu.pl 